\title{
Synchrotron Infrared Spectromicroscopy as a Novel Bioanalytical Microprobe for Individual Living Cells: Cytotoxicity Considerations
}

\author{
Hoi-Ying N. Holman, ${ }^{* 1}$ Kathleen A. Bjornstad, ${ }^{\dagger}$ Morgan P. McNamara, ${ }^{\dagger}$ \\ Michael C. Martin, ${ }^{\ddagger}$ Wayne R. McKinney, ${ }^{\ddagger}$ and Eleanor A. Blakely ${ }^{\dagger}$ \\ "Center for Environmental Biotechnology, ${ }^{\dagger}$ Life Sciences Division, ${ }^{\ddagger}$ Advanced Light \\ Source Division, Lawrence Berkeley National Lab, Berkeley, CA, 94720
}



\footnotetext{
${ }^{1}$ To whom correspondence and reprint requests should be addressed: Mail Stop 70A-3317, Lawrence Berkeley National Laboratory, 1 Cyclotron Road, Berkeley, CA 94720-0001. (510) 486-5943, HYHolman@1bl.gov
} 


\begin{abstract}
Synchrotron radiation-based Fourier transform infrared (SR-FTIR) spectromicroscopy is a newly emerging analytical tool capable of monitoring the biochemistry within an individual living mammalian cell in real time. This unique technique provides infrared (IR) spectra, hence chemical information, with high signal-to-noise at spatial resolutions as fine as 3 to 10 microns. Mid-IR photons are too low in energy $(0.05-0.5 \mathrm{eV})$ to either break bonds or to cause ionization, and the synchrotron IR beam has been shown to produce minimal sample heating. However, an important question remains, "Does the intense synchrotron beam induce any cytotoxic effects in living cells?" In this work, we present the results from a series of standard biological assays to evaluate any shortand/or long-term effects on cells exposed to the synchrotron radiation-based infrared (SR-IR) beam. Cell viability was tested using alcian blue dye-exclusion and colony formation assays. Cell-cycle progression was tested with bromodeoxyuridine (BrdU) uptake during DNA synthesis. Cell metabolism was tested using an MTT (3-[4,5Dimethylthiazol-2-yl]-2,5-diphenyltetrazolium bromide) assay. All control, 5-, 10-, and 20minute SR-IR exposure tests (267 total and over 1000 controls) show no evidence of cytotoxic effects. Concurrent infrared spectra obtained with each experiment confirm no detectable chemistry changes between control and exposed cells.
\end{abstract}

Keywords: Synchrotron; infrared; spectromicroscopy; FTIR; Cell viability; Cytotoxicity 


\section{INTRODUCTION}

Recent progress in analytical instrumentation has enabled dramatic advances in gene sequencing and protein identification techniques. Using the information produced by these techniques, the attention of biomedical researchers is now increasingly focused on understanding how chemical species interact in living organisms by the use of imaging techniques which simultaneously provide both morphological and chemical information within cells and tissues. Today intensive research in experimental biology, spectroscopy, and analytical instrumentation is seeking new ways to image chemical information within living cells. For correct elucidation of the cellular chemistry it is imperative to maintain the viability of the cell during and after the collection of the chemical image. Most imaging research has focused on the application of fluorescent probes that locate and identify a specific chemical event within the cell by the addition of a labeling compound. A major concern is the viability of the cell and possible biochemistry changes as a result of the monitoring and imaging process. To enhance viability by the reduction of bond breaking and ionization during fluorescent measurements, two-photon excitation at longer wavelengths has been used to lower the toxic effects of potentially bond-breaking UV or visible radiation on the sample. ${ }^{1-4}$ However, even this has recently been linked to apoptosis-like death. ${ }^{3}$

In contrast to fluorescent labeling techniques, synchrotron-based Fourier transform infrared (SR-FTIR) spectromicroscopy has the ability to monitor the chemistry occurring within an individual living cell without the need for labels and with even lower photon energies. Infrared (IR) spectroscopy is a sensitive analytical chemistry technique for studying biological systems. Many common biomolecules, such as nucleic acids, proteins, and lipids have characteristic and well-defined IR-active vibrational modes., Combining IR spectroscopy with microscopy yields a powerful tool for non-destructively 
probing bio-systems on a small size scale. ${ }^{7}$ With the recent addition of a synchrotron light source $e^{8-12}$ one can now obtain diffraction-limited spot sizes with high signal intensity in an IR microscope. This high brightness is of great advantage when measuring samples with a spatial resolution near the diffraction limit of 3 to 10 microns. The sample can be small and/or heterogeneous, for example; individual living cells, microorganisms, and larger biological systems in which local biochemistry may have significant spatial variations. Recent uses of synchrotron infrared spectromicroscopy include the examination of biological samples such as individual living cells, ${ }^{13-19}$ tissue samples, ${ }^{20-23}$ microbe-chemical interactions in environmental settings, ${ }^{24,25}$ protein conformations, ${ }^{26,27}$ and plant-soil interactions. ${ }^{28}$

It is crucial to know if the synchrotron radiation-based mid-infrared (SR-IR) source causes any short- or long-term effects on the living biological samples under study. Midinfrared photons are significantly lower in energy $(0.05-0.5 \mathrm{eV})$ than the excitation sources used in fluorescence probes including the newer two-photon techniques (photons energies of approximately $1 \mathrm{eV}$ ), implying that photo-induced effects will be minimal. However, to be assured that the SR-IR beam does not perturb living samples via other mechanisms, more detailed studies are required. SR-FTIR spectromicroscopy is an excellent tool for monitoring the biochemistry taking place within a cell. In all studies to date ${ }^{13-28}$ no changes have been observed in the IR spectra during the exposure to the synchrotron beam. This indicates no immediate changes in the overall biochemical composition of the exposed cell(s). Although mid-IR photons are too low in energy to directly break bonds or cause ionization, other effects from the SR-IR source may occur, including sample heating, drying, or other more subtle interactions which could influence long-term metabolic and other cellular physiological processes. We recently demonstrated that sample heating from the synchrotron IR beam is minimal $\left(\sim 0.5^{\circ} \mathrm{C}\right) .{ }^{29}$ 
The present paper presents the results of in vitro studies to determine if the SR-IR beam causes any detectable cytotoxic effects on living cells. Two classes of cyctotoxic effects were measured in this study: (1) immediate and/or short-term effects in cell viability, cell-cycle progression, cell metabolism, and (2) long-term effects on the proliferative/metabolic capacity of exposed cells. Four widely accepted cellular and molecular assays were selected to measure these potentially deleterious effects on cells subjected to different doses of the SR-IR beam. Finally, infrared spectra were also recorded for exposed and non-exposed cells, and were compared in detail to identify possible immediate chemical changes as a result of exposure to the SR-IR beam.

\section{MATERIALS AND METHODS}

Cells and cell synchronization. A human $\mathrm{T}-1$ cell-line from an established aneuploid cell-line derived from human kidney tissue was used in this study. ${ }^{30}$ They were selected partly because of their high plating efficiency (>90\%), and partly because they have been previously used as a model biological system in studies of the effects of radiation and oxygen on human cells. ${ }^{31-35}$ The T-1 cells were originally obtained from Dr. G.W. Barensen of the Radiobiological Institute of the Organization for Health Research, Rijswijk, The Netherlands, in 1976. They were maintained in growth medium consisting of Minimum Essential Medium (MEM) with Eagle's Balanced Salt Solution supplemented with $15 \%$ fetal calf serum (HyClone, Logan, Utah), $1 \mathrm{mM}$ L-glutamine, and pen strep antibiotics at $\mathrm{pH}$ 7.4. All chemicals were from Gibco/BRL unless noted. Cells were grown at $37^{\circ} \mathrm{C}$ in a humidified atmosphere of $5 \% \mathrm{CO}_{2}$ and $95 \%$ air. Cells were sub-cultured every 3-4 days.

Radiation effects on $\mathrm{T}-1$ cells are cell-cycle dependent, and are most damaging for $G_{1}$ and mitotic phase cells because their repair capacity is significantly less. ${ }^{32}$ Even within the $\mathrm{G}_{1}$-phase, the repair capacity has been reported to be negligible during the 
early $G_{1}$-phase, and improves as cells progress to the $G_{1} / S$ boundary ${ }^{32}$ In order to study a uniform $\mathrm{G}_{1}$-phase cell population, $\mathrm{T}-1$ cultures were grown to confluence to ensure para-synchronization. Immediately prior to each experiment cells were trypsinized from confluent cultures. Fluorescence-activated Cell Sorting (FACS) analysis demonstrated that $85 \%$ of the cells in these cultures were synchronized to $G_{0} / G_{1}$ phases. Cells were plated onto $60 \mathrm{~mm}$ petri dishes at a density of $1 \times 10^{2}$ cells/dish for the colony forming assay (see below), and $1 \times 10^{5}$ cells/dish for all other assays. Gold-coated glass microscope slide pieces $\left(0.25-\mathrm{cm}^{2}\right)$ were plated with $100 \mu \mathrm{l}$ of a $1 \times 10^{5}$ cells $/ \mathrm{ml}$ cell suspension for SR-FTIR measurements and confirmation of other assays. Cells were incubated in growth medium for two hours (just long enough for the cells to attach) in a $37^{\circ} \mathrm{C}$ and $5 \% \mathrm{CO}_{2}$ incubator before they were subjected to the tests detailed below.

Synchrotron infrared facility. This work was performed at the infrared spectromicroscopy beamline 1.4.3 at the Advanced Light Source (ALS) in Berkeley, CA. ${ }^{10-12}$ The synchrotron light is used as an external source for a Nicolet 760 FTIR bench and Nic-Plan ${ }^{\top M}$ IR microscope with a computer-controlled $x-y-z$ sample stage. The synchrotron infrared beam can be easily switched on and off via automated optics inside the microscope which can be controlled manually or by software. The ALS storage ring produces pulses of synchrotron light across a broad energy range from the far-IR to the hard x-ray. The front-end optics of BL 1.4.3 (bare aluminum coated optics and a CVDgrown diamond window) only pass photons with energies $<3 \mathrm{eV}$. The synchrotron light is then collimated and sent into the FTIR bench where it passes through a Ge coated $\mathrm{KBr}$ beamsplitter. This beamsplitter only allows light between $\sim 400$ and $\sim 10,000 \mathrm{~cm}^{-1}$ to continue on to the sample. Within this spectral bandwidth, we have measured an average power of $\sim 1 \mathrm{~mW}$ at the focused sample position within the IR microscope. The 
ALS light pulses are $40 \mathrm{ps}$ in duration with a $500 \mathrm{MHz}$ repetition rate. Therefore the peak power at the sample is 50 times higher than the average power, or $\sim 50 \mathrm{~mW}$. Conventional tungsten thermal IR sources, for example a Globar $^{\mathrm{TM}}$ source, provide, as measured in our instrument, approximately one half of the total average power over the same spectral bandwidth. The synchrotron infrared light is focused to a diffraction-limited (3 to 10 micron-diameter) spot, while the thermal light is focused into a spot of approximately 100 microns in diameter. This results in a brightness advantage of $\sim 150$ for the synchrotron source compared with the thermal source $\left(1.3 \times 10^{-2} \mathrm{~mW} / \mu \mathrm{m}^{2}\right.$ for the synchrotron versus $8.6 \times 10^{-5} \mathrm{~mW} / \mu \mathrm{m}^{2}$ for the thermal source).

A custom on-stage mini-incubator was used to maintain the proper moisture and growth environment for the cells while allowing in situ FTIR spectromicroscopy measurements. The mini-incubator was temperature controlled via circulating water from a water bath, and infrared transparent $\mathrm{CaF}_{2}$ windows on the top cover were separately temperature-controlled to avoid condensation. The location of the synchrotron infrared beam within the field of the microscope was fiducialized to approximately one micron by mapping a titanium on silicon calibration target. ${ }^{36}$

Exposure of individual cells to the synchrotron infrared beam. Immediately prior to synchrotron infrared exposure, the growth medium was replaced with fresh medium (100 $\mu \mathrm{l}$ per $60 \mathrm{~mm}$ dish, or $1 \mu \mathrm{l}$ per glass slide piece inside the humidified onstage mini-incubator as described earlier). Media plus $10 \mathrm{mM}$ Hepes was added to keep the cells hydrated, and the medium at $\mathrm{pH} 7.4$ throughout the exposure duration.

Every exposure experiment was conducted at $37^{\circ} \mathrm{C}$ and lasted for less than one hour. To ensure all test and control cells used in this study were synchronized (they were initially para-synchronized), we selected visually in the field of the infrared microscope 
only those single cells that had the shape and size typical of $\mathrm{G}_{1}$-phase $\mathrm{T}-1$ human cells. The selected cells were exposed to the focused synchrotron infrared beam for a specified duration of 5,10 , or 20 minutes. These times were chosen to greatly exceed the normal exposure time for data acquisition on an individual cell, typically between 20 seconds and 2 minutes. After the allotted time had passed, the beam was switched off, and the next test cell was located. Once completed, fresh growth media was replaced on the dish and it was returned to the standard $37^{\circ} \mathrm{C}$ and $5 \% \mathrm{CO}_{2}$ incubator.

Both negative and positive control cells were also tested. Negative controls were non-exposed cells located in the same field of the petri dish or gold-coated slide pieces (internal controls), and therefore experienced the same handling. Positive controls were cells that were either exposed to $70 \%$ alcohol solution, or allowed to progressively dehydrate as a result of evaporation of the growth medium at the edges of the samples under study.

Alcian blue assay for monitoring short-term effect on cell viability. The standard alcian blue dye-exclusion assay ${ }^{37}$ was used to detect if a cell remained viable and has intact membrane 6,12 , and 24 hours after its exposure to the synchrotron infrared beam. Viable cells are impermeable to the alcian blue molecules, whereas moribund cells are stained dark blue because of the breakdown in their membrane integrity.

This short-term assay relies on the active transport of alcian blue molecules back out of the living cell using energy derived from metabolism at the time of the assay. Test and control cells were incubated for six hours at $37^{\circ} \mathrm{C}$ and $5 \% \mathrm{CO}_{2}$ with growth medium and then fixed with $3 \%$ glutaraldehyde in Milliong's buffer, and $0.3 \%$ alcian blue (as stain) was added to give a final dye concentration of $0.03 \%$. Glutaraldehyde fixation was chosen because it can best preserve structural features of the cell membrane. ${ }^{37}$ 
Individual cells were then revisited with an optical microscope to determine the presence (or absence) of blue color -- blue indicates cell death.

Colony-forming assay to test long-term survival. In addition to the above dye exclusion method of short-term toxicity, we used the standard colony-forming assay ${ }^{31}$ to look for any potential effects of SR-IR beam on the long-term survival and proliferation on $\mathrm{T}-1$ cells. This assay began with plating cells at a low density of $\sim 100$ per $60 \mathrm{~mm}$ diameter petri dish to ensure resulting colonies would not overlap. Test cells were again identified visually in the field of the IR microscope according to their morphological characteristics including cell shape and cell size, and exposed by the synchrotron beam for the appropriate time of irradiation. The media was replaced and the dishes were returned to the standard $37^{\circ} \mathrm{C}$ and $5 \% \mathrm{CO}_{2}$ incubator and allowed to grow for 10 days.

Colony formation was determined by the following procedure. The cells were fixed and stained with $30 \%$ alcohol and $0.2 \%$ methylene blue at room temperature for one hour. The stain was then rinsed off, and the dishes were allowed to air dry. The sites of exposed and control cells were revisited under a microscope, and the number of cells that had grown for 10 days after the test event was recorded. Any cell that developed into a colony of at least fifty cells was considered to have maintained reproductive integrity. ${ }^{31}$ Any colony of less than fifty cells was considered to suffer from cytotoxic effects from the SR-IR beam. Resultant cell colonies from test and control cells were photographed in an optical microscope using fiduciary guides to relocate the original cells used for the SR-IR exposure. The reproductively proficient fraction of exposed and control cells were compared and the percent surviving fraction was calculated.

BrdU assay for the in situ detection of immediate cell-cycle progression effects. We evaluated the effect of SR-IR beam exposure on the cell-cycle progression by 
detecting when exposed and non-exposed T-1 cells reach their S-phase (DNA synthesis) after the release from confluence. The onset of DNA synthesis was detected in situ with the two-antibody BrdU assay according to the protocol (No. 1170 376) provided by Boehringer Mannheim. This standard assay relies on pulse-labeling the test and control cells with a final concentration of $10 \mu \mathrm{M}$ bromodeoxyuridine (BrdU) an hour before the estimated onset of DNA synthesis. BrdU, a thymidine analog, is known to be specifically incorporated into DNA during DNA synthesis. A conventional two-antibody method is used for identifying cells that have incorporated BrdU.

In our experiment BrdU was added to the growth medium eleven hours after cell release and ten hours post infrared exposure. They were incubated for one hour in the presence of $10 \mu \mathrm{M}$ BrdU (protected from light). Eleven hours plus one hour BrdU incubation was selected because previous studies of $\mathrm{T}-1$ cell cycling indicated that $\mathrm{T}-1$ cells are synthesizing DNA twelve hours into the cell cycle. ${ }^{33}$ We checked this timing by initiating BrdU uptake at 6,12 , or 24 hours after their release. We found indeed the majority (67\%) of our para-synchronized T-1 cells began synthesizing DNA at about 12 hours post-release, whereas less than $20 \%$ of cells began synthesizing DNA at 6 and 24 hours. Therefore all the reported BrdU assay results below were from tests with BrdU added 11 hours after cell setup. After a one-hour incubation in the BrdU-containing growth medium, cells were rinsed once with phosphate buffer saline (PBS) without calcium and magnesium, fixed with $-20^{\circ} \mathrm{C} 70 \%$ Ethanol, and air dried. Dishes with fixed cells on them were denatured by floating them on an $80^{\circ} \mathrm{C}$ waterbath for one minute in a fume hood with two $\mathrm{ml}$ of $70 \%$ formamide in a $2 \mathrm{X}$ saline-sodium citrate (SSC) buffer solution (Sigma) at $80^{\circ} \mathrm{C}$. Cells were then rinsed in PBS at room temperature, 
dehydrated for two minutes with $-20^{\circ} \mathrm{C} 70 \%$ ethanol followed by $-20^{\circ} \mathrm{C} 100 \%$ ethanol for two minutes, and dried.

After denaturing and alcohol dehydration, cells were rehydrated in $0.1 \%$ bovine serum albumin (BSA) blocking buffer in PBS. The primary mouse anti-BrdU antibody (Boehringer-Mannheim, Indianapolis, Indiana) was added at 1:20 in the BSA blocking buffer, protected from light, and incubated for one hour at room temperature. Cells were washed twice with PBS and then incubated with the secondary antibody, Alexa 594 (red) (Molecular Probe, Eugene, Oregon), diluted 1:100 in PBS for one hour. Cells were counter-stained with $0.25 \mu \mathrm{g} / \mathrm{ml}$ DNA-specific fluorochrome DAPI (4,6-diamidino-2phenylindole, from Sigma) for $5 \mathrm{~min}$ at room temperature, rinsed with PBS, and covered with Vectashield and a clean microscope slide coverslip. Individual cells were examined with a fluorescent microscope. Cells with red fluorescence in their nuclei (BrdU positive) were judged to be undergoing DNA synthesis. Those without red fluorescence were BrdU negative. The blue fluorescence of DAPI was used to identify the location of DNA in both BrdU-positive and BrdU-negative cells.

MTT assay to assess immediate metabolic activity effects. The extent of cellular metabolic activity (or biological redox reactions) in cells was detected in situ with the modified MTT technique according to the protocol (No. TOX-1, 7H258) provided with the assay kit (Sigma, St. Louis, Missouri). This modification of the protocol was eliminating the use of a UV/VIS spectrometer because our study requires the evaluation of individual cells.

The method relies on the ability of mitochondrial dehydrogenases produced in living cells to reduce the yellowish solution of tetrazolium salt (3-[4,5-Dimethylthiazol-2yl]-2,5-diphenyltetrazolium bromide) to an insoluble purple formazan. Test and control 
cells already subjected to 5-, 10-, and 20-minute IR exposures were incubated for 2 hours in medium containing tetrazolium salts at a final concentration of $1 \mathrm{mg} / \mathrm{ml}$ without phenol red. Cells were then revisited with an optical microscope. Cells that had a blue color were judged to have active mitochondrial dehydrogenases that reduce tetrazolium salt, and thus were metabolically active in this test. Cells that were not blue were considered metabolically inactive.

SR-FTIR spectromicroscopy to look for overall cellular chemical changes. We used SR-FTIR spectromicroscopy ${ }^{18}$ for detecting chemical vibrational mode changes in non-exposed controls and in cells exposed to the SR-IR beam. All SR-FTIR spectra were recorded in the $4000-600 \mathrm{~cm}^{-1}$ region as this mid-IR region contains unique molecular absorption fingerprints for cellular chemistry ${ }^{38}$. Because the $\mathrm{CaF}_{2}$ window of the on-stage mini-incubator does not transmit IR below $800 \mathrm{~cm}^{-1}$, some spectra were acquired by removing the $\mathrm{CaF}_{2}$ windows from the mini-incubator such that data extending to $600 \mathrm{~cm}^{-1}$ could be examined.

Every IR measurement consisted of 128 co-added spectra at a spectral resolution of $4 \mathrm{~cm}^{-1}$. All spectra were obtained in the double-pass transmission geometry and ratioed to a reference spectrum measured from a bare gold-coated slide, then absorbance values were computed. Residual water vapor and $\mathrm{CO}_{2}$ spectral features from imperfect purging of the spectrometer and IR microscope were subtracted using an appropriately scaled reference spectrum of air. The acquired spectra for the control and exposed cells were compared in detail to look for any spectral changes which could be caused by the exposure to the SR-IR beam. 


\section{RESULTS AND DISCUSSION}

Table 1 lists the number of individual cells tested by each cytotoxicity assay. Table 2 summarizes the analyzed results of these assays. For each biological assay we photographed the results for control and test cells that had been exposed to the SR-IR beam for 5-, 10-, or 20-minutes. Figures 1-4 show representative photographs for each assay. In no case did we find a result differing with the representative ones shown in the figures.

SR-IR beam has no short-term effect on cell viability. Alcian blue assays were carried out as described above. As shown in Figure 1, neither cells exposed to up to 20 minutes of synchrotron IR beam nor nearby non-exposed cells retained the blue dye 6 hours after exposure. This indicates that the SR-IR beam did not produce detectable effects on the viability of exposed cells. Other exposed cells remained free of stain 12 and 24 hours after exposure indicating that their membranes still remained intact. In contrast, dead positive control cells were stained blue as expected, as their membranes had become permeable to the dye molecules.

Cells survive and continue to proliferate days post exposure. While the aforementioned short-term test has revealed that cells were active at the time of the alcian blue assay, the long-term colony-forming assay demonstrates that the exposed cells also continue to proliferate into colonies. The exposed test cells and nearby nonexposed cells proliferated into colonies of similar size (Figure 2), well over fifty cells in ten days. The positive control cells, on the contrary, had detached from the petri dish and

disappeared from the field. Since none of the 46 SR-IR exposed test cells developed into colonies with less than 50 cells, we interpret this as an indication that SR-IR beam does not impact cell survival and proliferative activities. 
Exposure to SR-IR does not compromise cell-cycle progression. As described above the experiment was designed to specifically answer the question, "Do SR-IR beam exposed cells progress into S-phase at the same time as unexposed control cells?" Cellcycle progression in exposed cells were monitored by the incorporation of BrdU into newly synthesized DNA at 11 hours after cell setup and 10 hours post SR-IR exposure. Both exposed cells and non-exposed controls had reached the DNA synthetic phase (Sphase) of cell- cycle at this 12-hour observation point. For example, the upper photo in Figure 3 shows an image of 5-, 10-, or 20-minute exposed cells and their neighboring non-exposed controls. The lower photo in Figure 3 shows that all three exposed cells as well as the controls have incorporated BrdU into the DNA, which is identified by the double color labeling of red for BrdU and blue for DNA. The similarities among these immunofluorescent staining of BrdU (and DAPI) labeled cells indicate that the exposed cells are not compromised in their ability to enter their S-phase in the cell cycle after exposure to the SR-IR beam. Furthermore, the lack of detectable uptake of BrdU into DNA in exposed and control cells at 6 or 24 hours after their release demonstrates that the cell-cycle progression of SR-IR exposed cells remains uninterrupted.

ATP and $N A D^{+}$-associated metabolic activity is not impaired by the SR-IR beam. The two-hour MTT assay was carried out as described above on exposed, negative, and positive controls. Representative photos of the results are shown in Figure 4. Cells exposed for 20 minutes and nearby non-exposed controls show similar purple-blue stain. On the contrary, tetrazolium salt solution remained yellow in the killed (positive) controls with no purple-blue stain uptake. Results were identical for 5- and 10-minute exposures. This implies that both the exposed and negative control cells produced mitochondrial dehydrogenases during the two-hour MTT assay. Mitochondrial dehydrogenases are associated with the ubiquitous metabolic pathway of glycolysis ${ }^{39}$ that generates the 
critical biomolecules of ATP and NAD'. These results indicate that the SR-IR beam has negligible effects on this important metabolic pathway which provides energy to cells.

SR-FTIR spectra indicate no changes in chemistry inside exposed cells. Figure 5 shows spectra acquired for two nearby cells, one non-exposed and one that was exposed to the SR-IR beam for 30 minutes prior to data acquisition. The IR spectra were acquired using the synchrotron source for 70 seconds (128 co-added scans) as described earlier. The measured signal to noise ratio of the synchrotron IR spectra shown in Figure 5 is excellent, 1250:1. The two spectra are nearly identical with every peak position reproduced exactly and only slight overall intensity variations due to a small difference in the thickness of the two cells. These results indicate that there are no chemical changes happening in the cell upon exposure to the SR-IR beam. We also performed IR measurements repeatedly on one living cell every 10 minutes for thirty minutes and the IR spectrum remained unchanged to within 0.005 absorbance units across the entire mid-IR spectral range. Longer exposure times can be tested, however the living cell continues growing through its cell cycle, which results in other previously reported spectral changes. ${ }^{19}$

In all 5 assays studied (alcian blue, colony formation, BrdU, MTT, and SR-IR spectra) we found no detectable changes between cells exposed for 5,10 , and 20 minutes to the synchrotron infrared beam and nearby non-exposed controls. 267 individual cells were tested using standard biochemical assays with zero tests showing any measurable cytotoxic effects (counting statistics error is 6.1\%), with over 1000 control cells used. Additionally, infrared spectra that are a measure of the overall biochemistry within a cell were obtained from test and control cells, and showed no spectral changes. These results show that the high-brightness mid-IR synchrotron beam 
is not only non-destructive, but also causes no effects on both the short- and long-term viability, proliferation, and metabolism within living human T-1 cells. Although the present study has focused on only one established human cell line, we anticipate that these results will be generally applicable to most, if not all, living biological systems.

The results reported here lay an important foundation for future biomedical and biological applications of synchrotron infrared spectromicroscopy, which will complement other biochemistry and microscopy techniques. SR-FTIR spectromicroscopy enables the successive monitoring of biochemical changes in individual cells non-destructively without having to treat cells with exogenous dyes, fluorescent labels, or stains, or to resort to destructive techniques. The non-invasive and non-destructive nature of the technique allows each cell in a population to be studied sequentially over a period extending to hours or even days. By monitoring individual cells over time it will be possible to detect the onset of disease and other cellular changes, and to probe the heterogeneity of responses to various treatments or insults within a population of living cells. The development of SR-FTIR spectromicroscopy will result in a broadly applicable and powerful research tool available to the scientific community.

\section{ACKNOWLEDGEMENTS}

We thank Kelly Knutsen and Richard Saykally for assistance with the synchrotron beam power measurements. This research was supported by the Office of Science, Office of Biological and Environmental Research, Medical Science Division and the Office of Science, Office of Basic Energy Sciences, Materials Sciences Division, of the U.S. Department of Energy under Contract No. DE-AC03-76SF00098 at Lawrence Berkeley National Laboratory. 


\section{REFERENCES}

${ }^{1}$ A. Diaspro, "Introduction to two-photon microscopy," Microscopy Research and Technique 47 (3), 163-164 (1999).

${ }^{2}$ K. Konig, "Multiphoton microscopy in life sciences," Journal of Microscopy-Oxford 200 (PT2), 83-104 (2000).

${ }^{3}$ U.K. Tirlapur, K. Koenig, C. Peuckert et al., "Femtosecond near-infrared laser pulses elicit generation of reactive oxygen species in mammalian cells leading to apoptosis-like death," Experimental Cell Research 263 (1), 88-97. (2001).

${ }^{4}$ J.M. Squirrell, D.L. Wokosin, J.G. White et al., "Long-term two-photon fluorescence imaging of mammalian embryos without compromising viability," Nature Biotechnology 17 (8), 763-767. (1999).

${ }^{5}$ F.S. Parker, Applications of infrared spectroscopy in biochemistry, biology, and medicine (Plenum Press, New York, 1971).

${ }^{6}$ H.H. Mantsch and D. Chapman, Infrared spectroscopy of biomolecules (Wiley-Liss, New York, 1996).

${ }^{7}$ M. Diem, S. Boydston-White, and L. Chiriboga, "Infrared spectroscopy of cells and tissues: Shining light onto a novel subject," Applied Spectroscopy 53 (4), 148A-161A. (1999).

${ }^{8}$ J.A. Reffner, P.A. Martoglio, and G.P. Williams, "Fourier Transform Infrared Microscopical Analysis with Synchrotron Radiation - the Microscope Optics and System Performance," Review of Scientific Instruments 66 (2), 1298-1302 (1995).

${ }^{9}$ G.L. Carr, J.A. Reffner, and G.P. Williams, "Performance of an infrared microspectrometer at the NSLS," Review of Scientific Instruments 66 (2), 1490-2 (1995). 
${ }^{10}$ W.R. McKinney, C.J. Hirschmugl, H.A. Padmore et al., "The first infrared beamline at the ALS: design, construction, and initial commissioning," presented at the AcceleratorBased Infrared Sources and Applications, San Diego, CA, 1997 (unpublished).

${ }^{11}$ M.C. Martin and W.R. McKinney, "The first synchrotron infrared beamlines at the Advanced Light Source: microspectroscopy and fast timing," presented at the Applications of Synchrotron Radiation Techniques to Materials Science IV, Materials Research Society, 1998 (unpublished).

${ }^{12}$ W.R. McKinney, M.C. Martin, J.M. Byrd et al., "First infrared beamlines at the ALS: final commissioning and new end stations," presented at the Accelerator-based Sources of Infrared and Spectroscopic Applications, Denver, CO, 1999 (unpublished).

${ }^{13}$ D.L. Wetzel, J.A. Reffner, and G.P. Williams, "Synchrotron-powered FT-IR microspectroscopy: Single cell interrogation," Mikrochimica Acta (SUPP14), 353-355 (1997).

${ }^{14}$ L.M. Miller, G.L. Carr, G.P. Williams et al., "Synchrotron infrared microspectroscopy as a means of studying chemical composition at a cellular level," Biophysical Journal 72 (2 PART 2), A214. (1997).

${ }^{15} \mathrm{~N}$. Jamin, P. Dumas, J. Moncuit et al., "Highly resolved chemical imaging of living cells by using synchrotron infrared microspectrometry," Proceedings of the National Academy of Sciences of the United States of America 95 (9), 4837-4840. (1998).

${ }^{16} \mathrm{~N}$. Jamin, P. Dumas, J. Moncuit et al., "Chemical imaging of nucleic acids, proteins and lipids of a single living cell. Application of synchrotron infrared microspectrometry in cell biology," Cellular and Molecular Biology 44 (1), 9-13 (1998).

${ }^{17}$ H.-Y.N. Holman, M. Zhang, R. Goth-Goldstein et al., "Detecting exposure to environmental organic toxins in individual cells: toward development of a microfabricated device," in Micro- and Nanofabricated Structures and Devices for Biomedical 
Environmental Applications II, edited by M. Ferrari (SPIE Proceedings, Bellingham, WA, 1999), Vol. 3606, pp. 55-63.

${ }^{18}$ H.-Y.N. Holman, R. Goth-Goldstein, M.C. Martin et al., "Low-dose responses to 2,3,7,8tetrachlorodibenzo-p-dioxin in single living human cells measured by synchrotron infrared spectromicroscopy," Environmental Science and Technology 34 (12), 2513-2517 (2000). ${ }^{19}$ H.-Y.N. Holman, M.C. Martin, E.A. Blakely et al., "IR spectroscopic characteristics of cell cycle and cell death probed by synchrotron radiation based Fourier transform IR spectromicroscopy," Biopolymers: Biospectroscopy 57 (6), 329-335 (2000).

${ }^{20}$ L.-P.I. Choo, D.L. Wetzel, W.C. Halliday et al., "In situ characterization of beta-amyloid in Alzheimer's diseased tissue by synchrotron fourier transform infrared microspectroscopy," Biophysical Journal 71 (4), 1672-1679. (1996).

${ }^{21}$ L.M. Miller, C.S. Carlson, G.L. Carr et al., "A method for examining the chemical basis for bone disease: Synchrotron infrared microspectroscopy," Cellular and Molecular Biology 44 (1), 117-127 (1998).

${ }^{22} \mathrm{~F}$. Briki, B. Busson, L. Kreplak et al., "Exploring a biological tissue from atomic to macroscopic scale using synchrotron radiation: Example of hair," Cellular and Molecular Biology 46 (5), 1005-1016 (2000).

${ }^{23}$ L.M. Miller, J. Tibrewala, and C.S. Carlson, "Examination of bone chemical composition in osteoporosis using fluorescence-assisted synchrotron infrared microspectroscopy," Cellular and Molecular Biology 46 (6), 1035-1044 (2000).

${ }^{24}$ H.-Y.N. Holman, D.L. Perry, M.C. Martin et al., "Real-time Characterization of Biogeochemical Reduction of $\mathrm{Cr}(\mathrm{VI})$ on Basalt Surfaces by SR-FTIR Imaging," Geomicrobiology Journal 16 (4), 307-324 (1999). 
${ }^{25}$ J.T. Geller, H.Y. Holman, G. Su et al., "Flow dynamics and potential for biodegradation of organic contaminants in fractured rock vadose zones," Journal of Contaminant Hydrology 43 (1), 63-90 (2000).

${ }^{26}$ L.M. Miller, A.J. Pedraza, and M.R. Chance, "Identification of conformational substates involved in nitric oxide binding to ferric and ferrous myoglobin through difference Fourier transform infrared spectroscopy (FTIR)," Biochemistry 36 (40), 12199-12207 (1997).

${ }^{27}$ A.H. Xie, Q. He, L. Miller et al., "Low frequency vibrations of amino acid homopolymers observed by synchrotron far-IR absorption spectroscopy: Excited state effects dominate the temperature dependence of the spectra," Biopolymers 49 (7), 591-603 (1999).

${ }^{28}$ T.K. Raab and M.C. Martin, "Visualizing rhizosphere chemistry of legumes with midinfrared synchrotron radiation," Planta 212, In Press (2001).

${ }^{29}$ M.C. Martin, N.M. Tsvetkova, J.H. Crowe et al., "Negligible sample heating from synchrotron infrared beam," Applied Spectroscopy 55 (2), 111-113 (2001).

${ }^{30} \mathrm{~J}$. van der Veen, L. Bots, and A. Mes, "Establishment of Two Human Cell Strains from Kidney Reticulosarcoma of Lung," Arch. Ges. Virusforsch 8, 230-238 (1958).

${ }^{31}$ G.W. Barendsen, C.J. Koot, G.R. Van Kersen et al., "The effect of oxygen on impairment of the proliferative capacity of human cells in culture by ionizing radiations of different LET," International Journal of Radiation Biology and Related Studies in Physics, Chemistry and Medicine 10 (4), 317-27 (1966).

${ }^{32}$ E.A. Blakely, P.Y. Chang, and L. Lommel, "Cell-Cycle-dependent Recovery from Heavy-Ion Damage in G-1-phase Cells," Radiation Research 104 (2 PART 2), S-145-S157 (1985). 
${ }^{33}$ E.A. Blakely, R.J. Roots, P.Y. Chang et al., "Cell-Cycle Dependence of X-Ray Oxygen Effect Role of Endogenous Glutathione," $\mathrm{NCl}$ (National Cancer Institute) Monographs (6), 217-224 (1988).

${ }^{34}$ E.A. Blakely, "Cell inactivation by heavy charged particles," Radiation and Environmental Biophysics 31 (3), 181-196 (1992).

${ }^{35}$ E.A. Blakely, C.A. Tobias, T.C. Yang et al., "Inactivation of human kidney cells by highenergy monoenergetic heavy-ion beams," Radiation Research 80 (1), 122-60 (1979).

${ }^{36}$ A.C. Thompson, J.H. Underwood, E.H. Anderson et al., "Characterization of the focal quality of micron-size beams from x-ray mirrors and zone plates," presented at the Advances in X-Ray Optics, San Diego, CA, 2000 (unpublished).

${ }^{37}$ D.K. Yip and N. Auersperg, "The dye-exclusion test for cell viability: persistence of differential staining following fixation," In Vitro 7 (5), 323-9 (1972).

${ }^{38} \mathrm{~J}$. Liquier and E. Taillandier, "Infrared spectroscopy of nucleic acids," in Infrared Spectroscopy of Biomolecules, edited by H.H. Mantsch and D. Chapman (Wiley-Liss, New York, 1996), pp. 131-158.

${ }^{39}$ T.F. Slater, Biochim. Biophys. Acta 77, 383 (1963). 
Table 1. Summary showing the numbers of synchrotron IR exposure tests conducted on individual living cells with each cytotoxicity assay. Neighboring non-exposed cells were used as negative controls. A total of 267 SR-IR exposed cells were studied, with over 1000 control cells used.

\begin{tabular}{|l|c|c|c|c|}
\hline & $\begin{array}{c}\text { Alcian } \\
\text { Blue (AB) } \\
\text { Assay }\end{array}$ & $\begin{array}{c}\text { Colony } \\
\text { Formation } \\
\text { Assay } \\
\text { (CFA) }\end{array}$ & $\begin{array}{c}\text { BrdU } \\
\text { Assay }\end{array}$ & $\begin{array}{c}\text { MTT } \\
\text { Assay }\end{array}$ \\
\hline Non-exposed controls & $>200$ & $>180$ & $>350$ & $>300$ \\
\hline 5-minute exposure & 20 & 12 & 31 & 23 \\
\hline 10-minute exposure & 19 & 12 & 21 & 23 \\
\hline 20-minute exposure & 20 & 22 & 35 & 29 \\
\hline
\end{tabular}


Table 2. A summary of results from the cytotoxicity assays for SR-IR exposed cells compared to negative and positive controls.

\begin{tabular}{|l|c|c|c|c|}
\hline & $\begin{array}{c}\text { AB Assay } \\
\text { (\% of cells } \\
\text { excreting } \\
\text { dye) }\end{array}$ & $\begin{array}{c}\text { CFA } \\
\text { (\% of cells } \\
\text { forming } \\
\text { colonies) }\end{array}$ & $\begin{array}{c}\text { BrdU } \\
\text { Assay } \\
\text { (\% of } \\
\text { cells at } \\
\text { S-phase) }\end{array}$ & $\begin{array}{c}\text { MTT } \\
\text { Assay } \\
\text { (\% of cells } \\
\text { reducing } \\
\text { MTT) }\end{array}$ \\
\hline Exposed Cells & 100 & 100 & 100 & 100 \\
\hline Non-exposed (Negative) Controls & 100 & 100 & 100 & 100 \\
\hline Killed (Positive) Controls & 0 & 0 & - & 0 \\
\hline
\end{tabular}




\section{Figure Captions:}

Figure 1. Photograph showing results from alcian blue assays of cells exposed to the SR-IR beam for (a) 5 minutes, (b and c) 10 minutes, and (d) 20 minutes. Other cells in the field were not exposed and were used as negative controls. No cells show retention of the blue dye demonstrating that no immediate cytotoxicity is observed.

Figure 2. Photographs showing typical results for colony forming from (a) a negative control cell and (b) a test cell that had been exposed to the SR-IR beam for 20 minutes. Both cells proliferated into similar sized colonies after 10 days.

Figure 3. Photographs showing BrdU assay results for cells exposed to the SR-IR beam for (a) 5 minutes, (b) 10 minutes, and (c) 20 minutes. Two other cells in the field were unexposed and used as negative controls. In the lower panel, the blue color indicates DNA and the red color indicates BrdU incorporation during DNA synthesis. All test and control cells show the same incorporation of BrdU into the DNA.

Figure 4. Photograph showing typical MTT assay results for control and test cells that had been exposed to the SR-IR beam for (a) 5 minutes, (b) 10 minutes, and (c) 20 minutes. Other cells in the field were unexposed and are used as controls. All test and control cells show the same blue color indicating the same level of metabolic activity.

Figure 5. Infrared spectra for a control cell (black line) and a cell exposed to the SR-IR beam for 30 minutes (red line). The small intensity variation between the two spectra is due to a slight difference in thickness between the two cells. Otherwise the spectra are very much the same indicating no overall change in biochemistry has occured. 


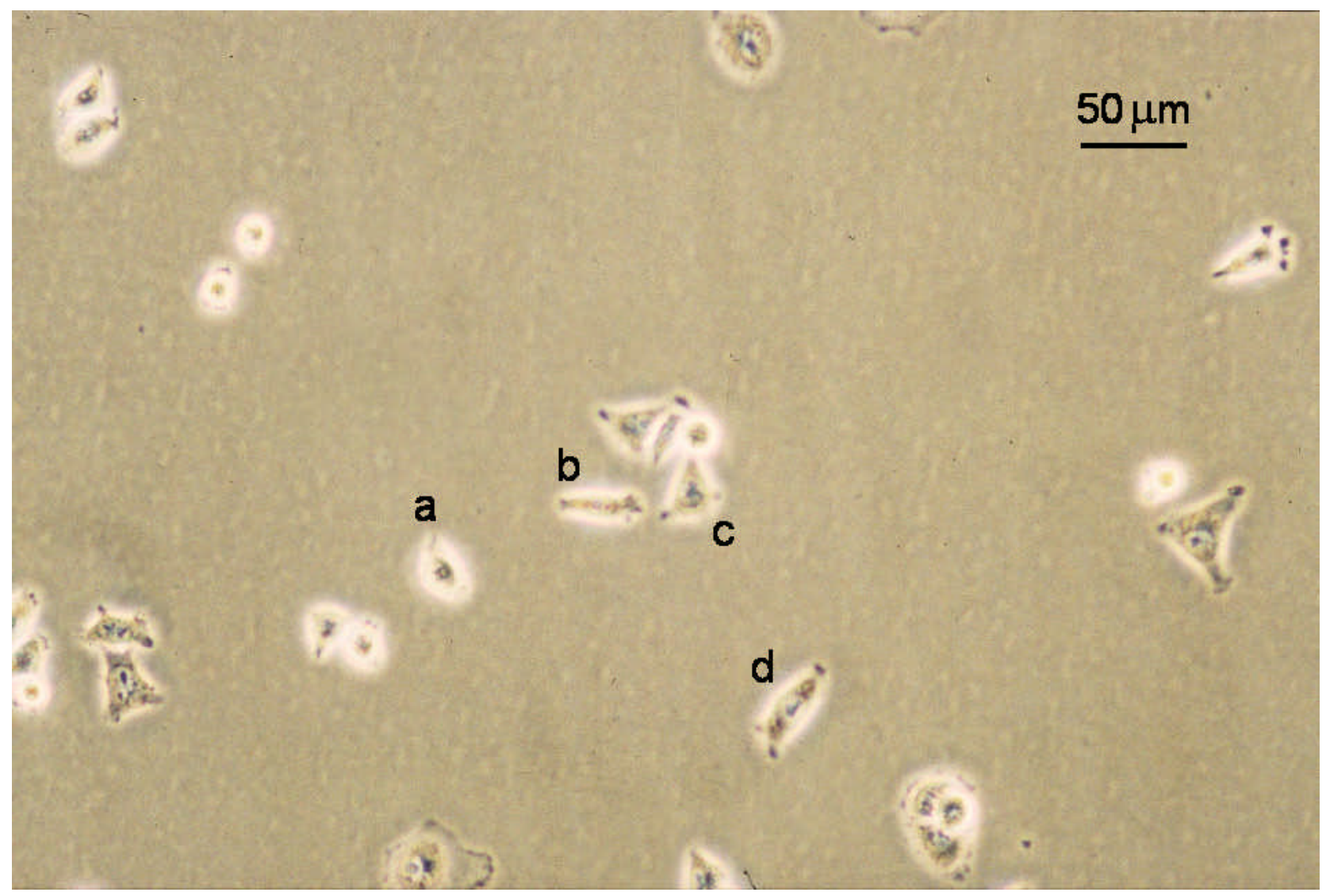

Holman et al., Figure 1. 


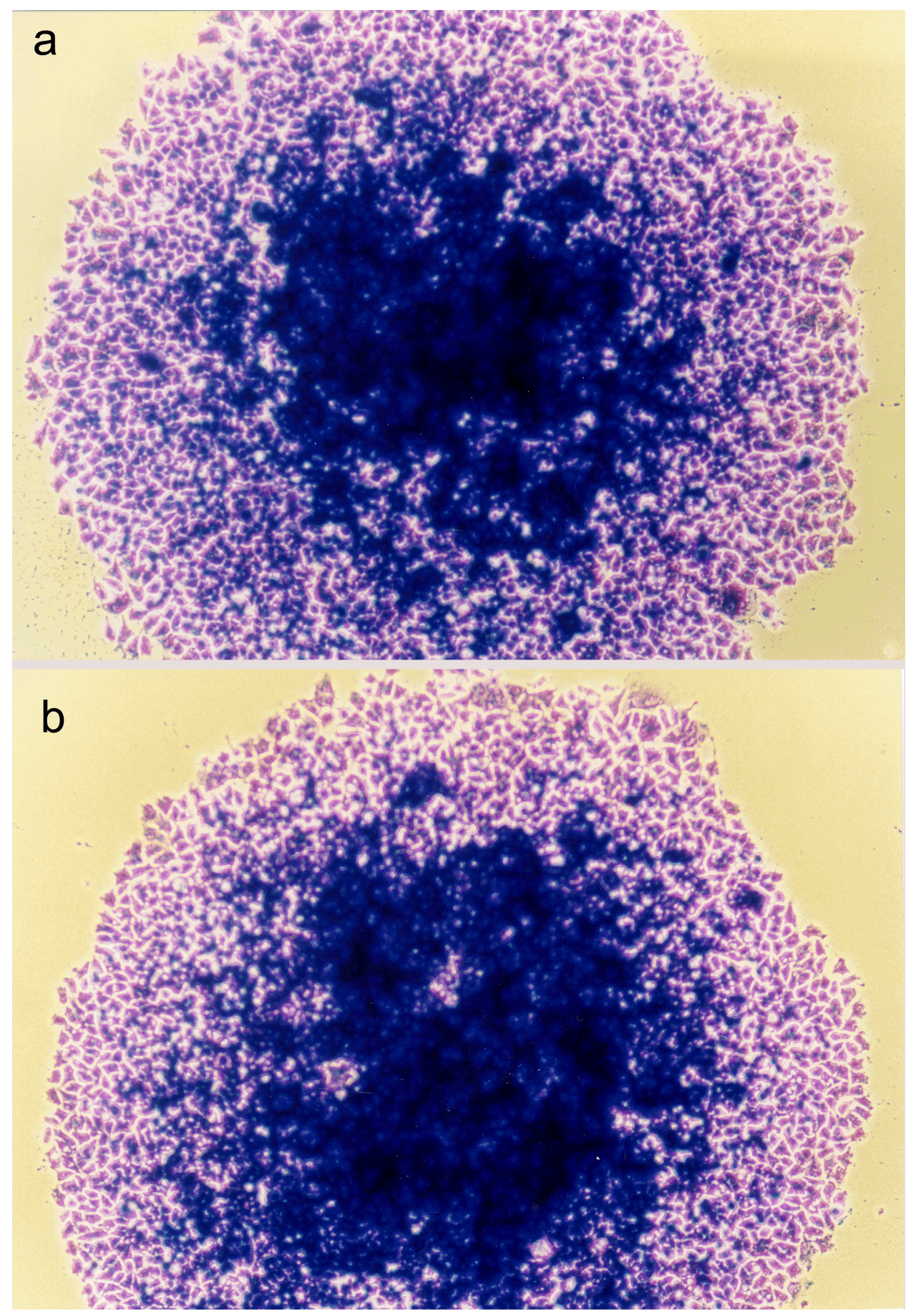

Holman et al., Figure 2. 


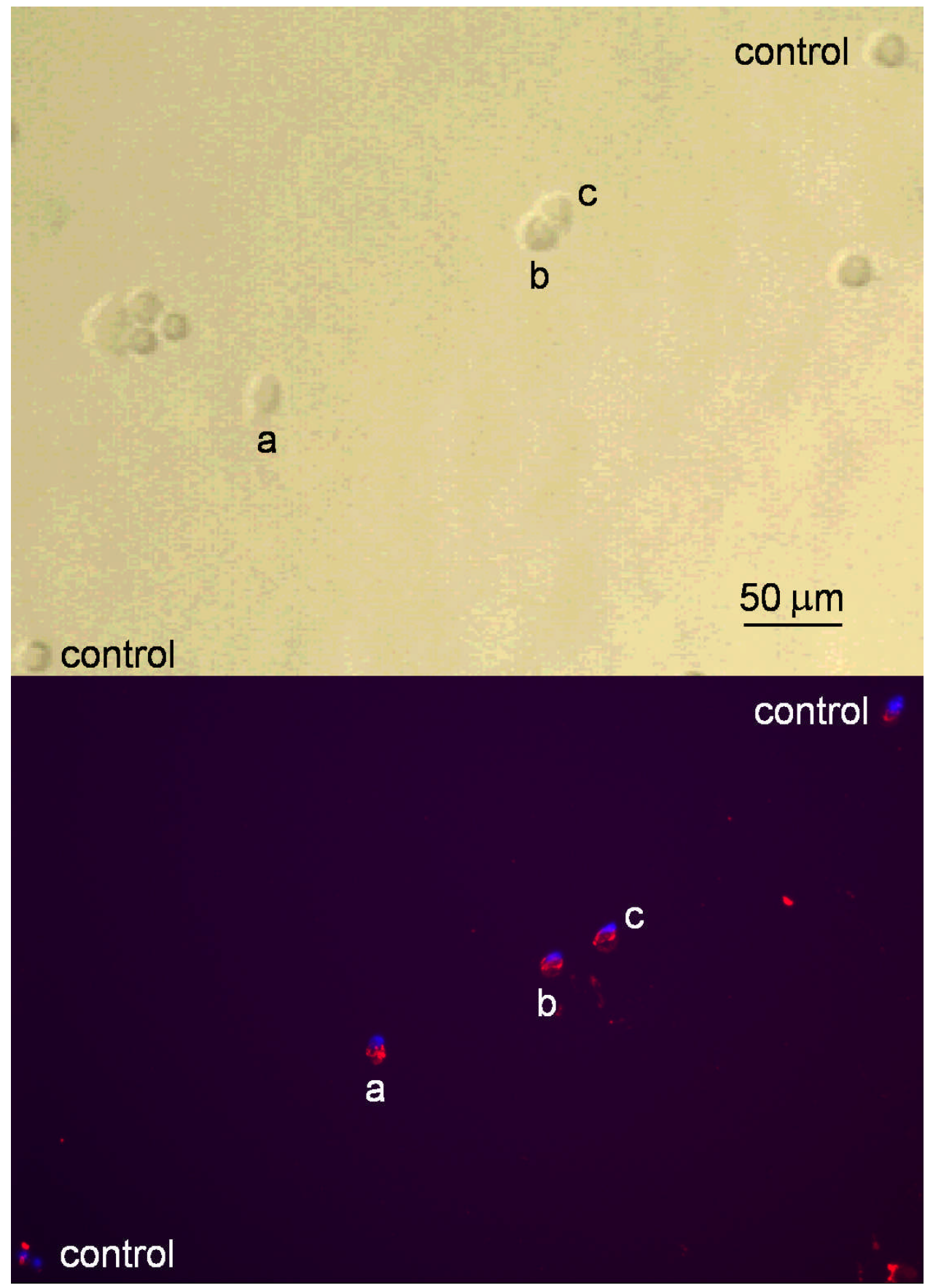

Holman et al., Figure 3. 


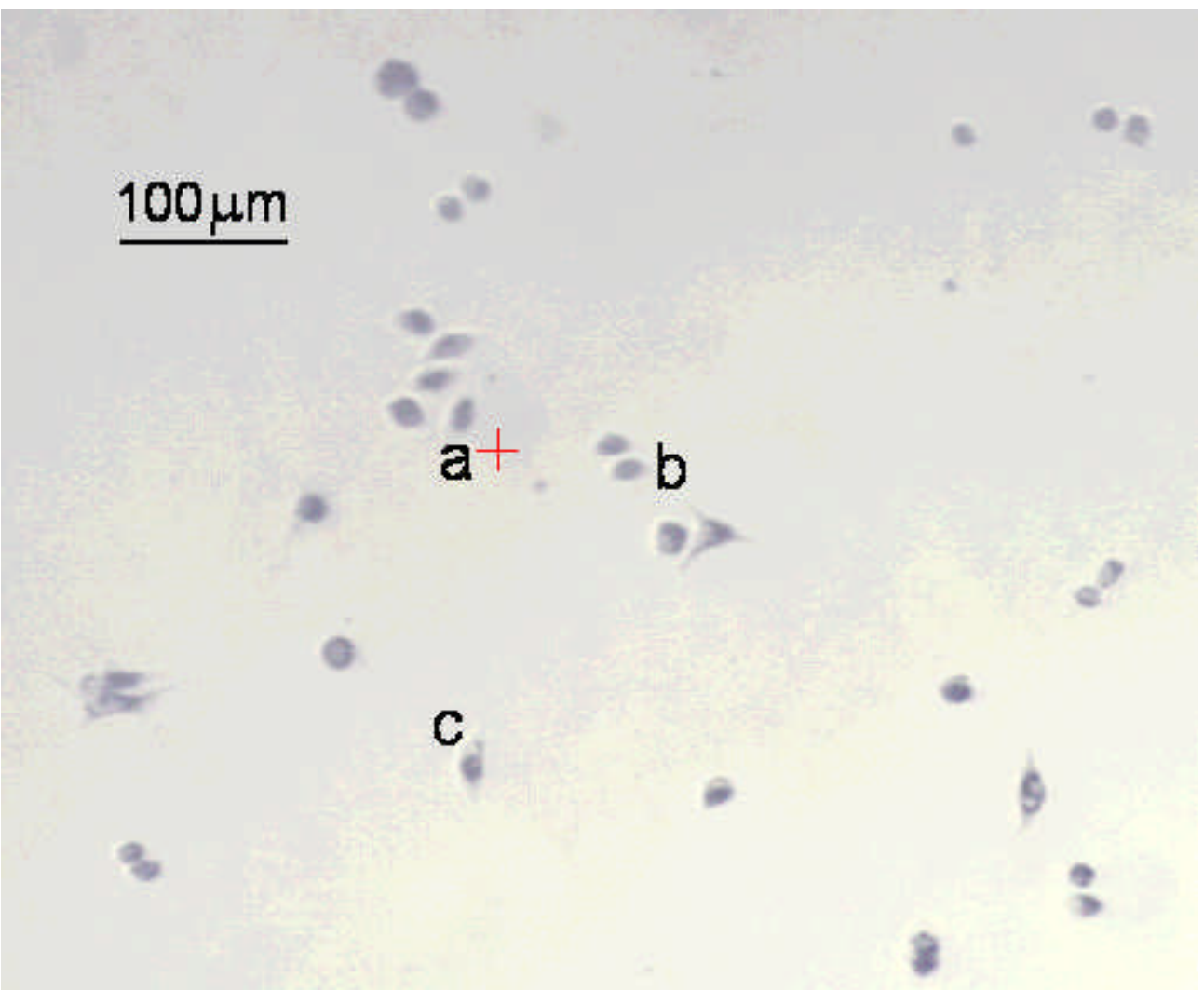

Holman et al., Figure 4. 




Holman et al., Figure 5. 